\title{
Improving the Performance of On-Board Cache for Flash-based Solid-State Drives
}

\author{
Miaoqing Huang, Liang Men \\ Department of Computer Science and Computer Engineering, University of Arkansas \\ Fayetteville, Arkansas 72701, USA \\ Email: \{mqhuang,mliang\}@uark.edu
}

\begin{abstract}
Flash-based Solid-State Drives (SSDs) are data storage devices that use flash memory to store persistent data. Previously we presented a centralized on-board cache for SSDs to improve the response time and reduce the physical writes to the flash media. An automatic periodic update (APU) feature with fixed period is used to write dirty and stable cache lines to flash media when the SSD is idle. In this work we propose a distributed on-board cache architecture to match the intrinsic parallelism of SSDs. Since there are $N$ individual flash packages in one SSD device, $N$ dirty cache lines are selected by the APU feature, each of which is written to a separate flash package. Simulation results show that this distributed on-board cache can significantly reduce the response time of SSDs by up to $\mathbf{9 0 \%}$ compared with the SSDs without such a cache, while reducing the number of physical writes to the flash memory.
\end{abstract}

Keywords-solid-state drives; automatic periodic update; performance improvement; simulation.

\section{INTRODUCTION AND BACKGROUND}

Flash-based solid-state drives (SSDs) exhibit significantly faster read access times compared with hard disk drives (HDDs), but suffer from write speeds that are generally inferior to that of HDDs. As a result, there is a large imbalance between the speeds of SSDs for read and write. It is therefore desirable that the on-board cache on data storage drives balances out the disparity between reads and writes, in addition to improving the average read/write access time. In our previous work [1] a centralized on-board cache was proposed, as shown in Figure 1. The basic cache policy is write back, i.e., write requests are always serviced by the cache. Thanks to the superior read performance of SSDs, read requests are not directly serviced by the cache unless the corresponding data already exist in the cache due to previous write requests. In the write-back cache, write misses to dirty cache lines will trigger a cache line eviction, which will flush the old cache line to flash memory. This data flush is termed as "on-demand update" in this work. Ondemand update will take place only when a write miss occurs and there is no stale/clean cache lines. The least recently used (LRU) policy is used to select a dirty cache line in a $p$ way set to evict. In addition to the manual eviction of cache lines due to write misses, an automatic periodic update (APU) feature was presented. The APU feature writes back stable but dirty cache lines according to a predetermined set of policies during the idle time of the SSD device. Different policies such as APU-LRU and APU-LFU can be applied for selecting the appropriate cache line to flush. In APULRU, a dirty cache line with the least recent access time is selected to evict. On the other hand, a dirty cache line with the smallest access frequency is selected when applying APU-LFU policy. Please note that all dirty lines in the whole cache are candidates for a possible flush to flash memory during the automatic update process.

The write-back-only cache in the SSD may introduce the data integrity issue. Without automatic update, the dirty cache lines will stay dirty. Although these dirty cache lines can be updated to flash media when the computer is turned off, a sudden power loss or an OS crash can introduce data integrity issue. On the other hand, the automatic periodic update feature will write those dirty cache lines to the flash media in a short amount of time. For example, it takes less than 6 seconds to write all contents of a $64 \mathrm{MB}$ cache into flash media. This APU feature will largely resolve the data integrity issue for using the on-board cache.

The internal architecture of SSD provides more potential for the on-board cache. An SSD typically consists of multiple flash packages, which can be accessed in parallel. Therefore in this work we propose a distributed cache design to take the advantage of this intrinsic parallelism. Two different mechanisms can be adopted to implement this approach. (1) The on-board cache can be physically broken into independent pieces of equal size. Depending on the number of these small caches, each cache will service the $\mathrm{read} / \mathrm{write}$ requests of one or more flash packages. (2) The on-board cache is still shared by all flash packages. However, during the automatic update period, multiple dirty cache lines are written into the flash packages simultaneously. Experimental results show that the second mechanism is more efficient than the first one for overall performance.

\section{Distributed ON-BOARD CACHE}

As shown in Figure 1(a) there are multiple flash packages in a single SSD. These flash packages can be accessed in parallel. In our previous design, there is only one centralized cache. The cache manager will monitor the access requests to the cache. Once there is no access request in a certain period of time, e.g., $2 \mathrm{~ms}$, the cache manager will start flushing the content of dirty cache lines into the flash memory until one access request arrives. During the flushing period, only one dirty cache line is flushed at a time. Since only one 


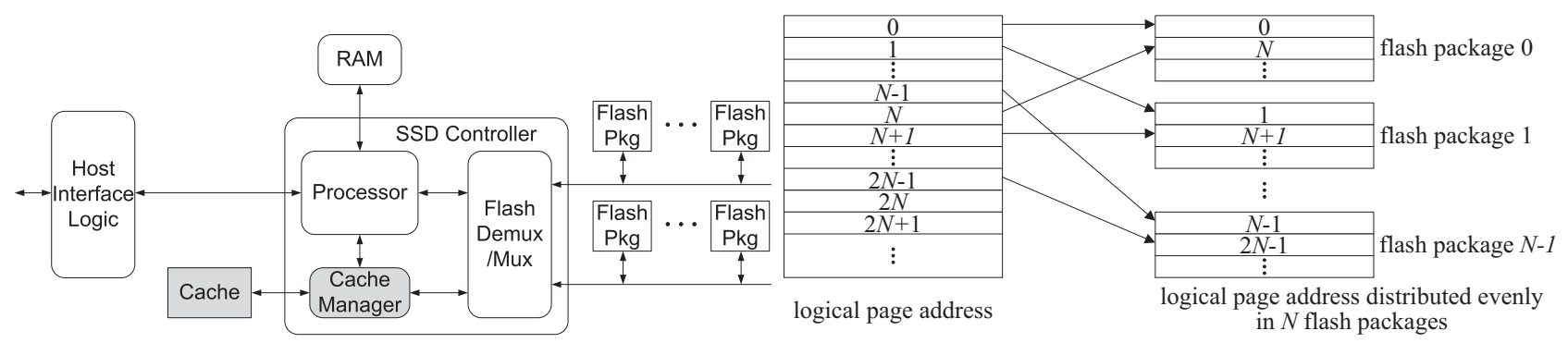

(a) Internal architecture of an SSD with on-board cache [2].

(b) Logical flash page addresses distribution.

Figure 1. Internal architecture of an SSD device.

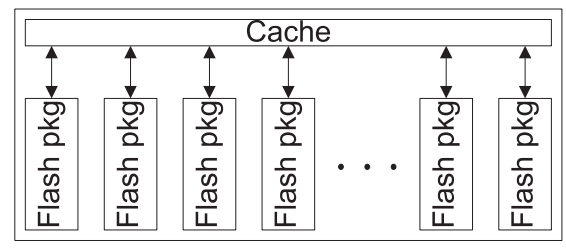

(a) 1 (cache) : $N$ (flash packages).

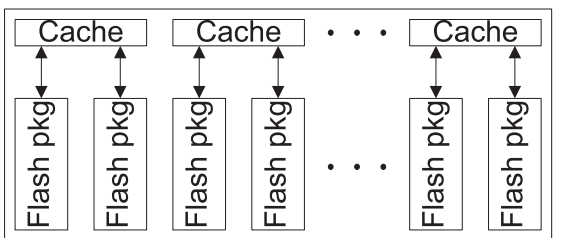

(b) $1: 2$.

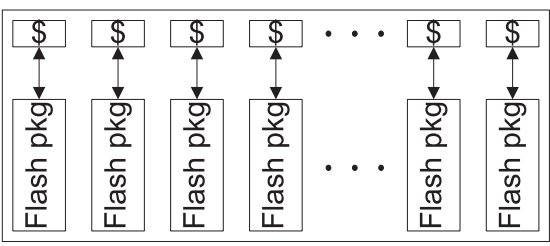

(c) $1: 1$.

Figure 2. Various configurations of distributed cache designs.

flash package is accessed at a time, the write bandwidth of other flash packages is wasted. A distributed cache can fully utilize the write bandwidth by writing multiple cache lines to these flash packages in parallel. Figure 2 illustrates the various options to provide a distributed view of the cache.

$\checkmark 1$ (cache) : $N$ (flash packages), in which ' $N$ ' is the total number of flash packages in an SSD device, as shown in Figure 2(a). In this case, all the data access requests to the whole $\mathrm{SSD}$ device are serviced by the single cache. During the APU period, $N$ dirty cache lines are selected simultaneously, one cache line for each flash package. Although the cache is still a central one, it can be considered as a logically distributed cache.

- 1: $m$ where $1<m<N$. The total size of the cache is evenly divided such that $m$ flash packages share a single piece, as shown in Figure 2(b) in which $m=2$. In this case, the cache is physically distributed. However, $m$ flash packages share a single cache. The data access requests for the $m$ flash packages are serviced by the same cache. During the APU period, $m$ dirty cache lines are selected in parallel for those flash packages that share the same cache.

$\checkmark$ 1:1. The total size of the cache is evenly divided such that one flash package has its own cache, as shown in Figure 2(c).

These different configurations have their own advantages and disadvantages. The 1:N configuration may provide the overall small conflict rate; however, the write requests to different flash pages may step on each other. The 1:1 configuration provides the best isolation. But the conflict rate may be very high. The $1: m$ configuration provides the compromise between the two extremes.
The implementation of cache management for these different types of distributed cache is subject to the translation between the logical addresses and the physical addresses in the flash translation layer (FTL). For the SSD module used in our simulation, the continuous logical flash page addresses are evenly distributed into $N$ flash packages as shown in Figure 1(b). Therefore, continuous logical addresses are always distributed to different flash packages. Inside each flash page, there is an internal mapping table between the logical address and physical address at the page level. Consecutive write requests to the same flash package are serviced by continuous flash pages in the same block until the block becomes full, in which case a new blank block is picked to serve the write requests.

The proposed cache design is simulated using DiskSim [3], which provides a simulation infrastructure for data storage devices and systems. An SSD model developed by Microsoft Research [2] was modified to include the proposed cache model, and then incorporated into DiskSim.

\section{A. Simulation Setup}

In order to demonstrate the effectiveness of the on-board cache, two different sets of benchmarks, microbenchmark and real applications, are simulated on two different SSD implementations, i.e., SSD with/without cache. For the case using the proposed cache, six different sub-cases are considered, as follows.

- Write Back: basic write-back strategy without APU.

- APU-LRU: write-back cache with APU applying LRU algorithm. Only one cache line is selected each time. 


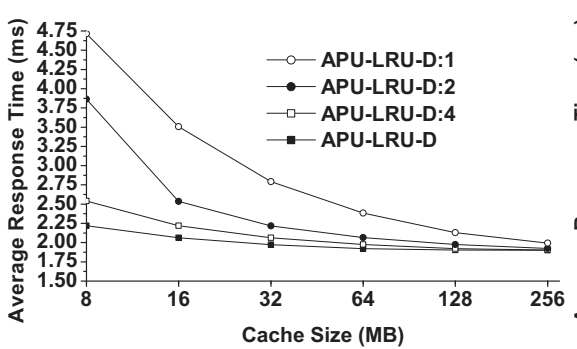

(a) Microbenchmark: direct mapped.

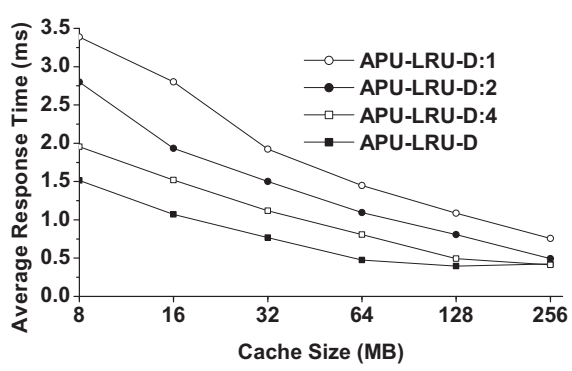

(d) IOzone: direct mapped.

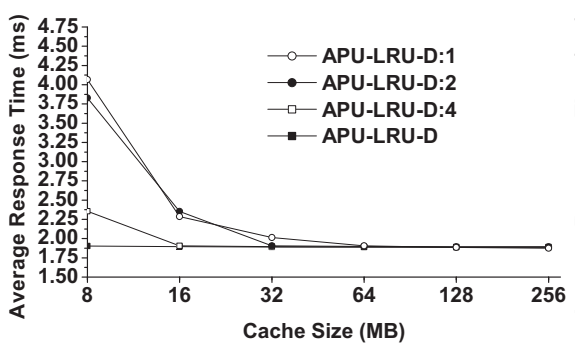

(b) Microbenchmark: 2-way set associative.

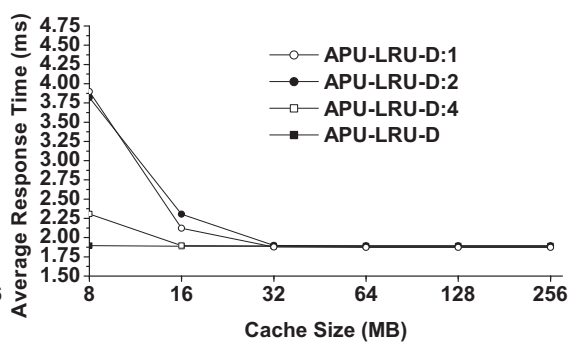

(c) Microbenchmark: 4-way set associative.

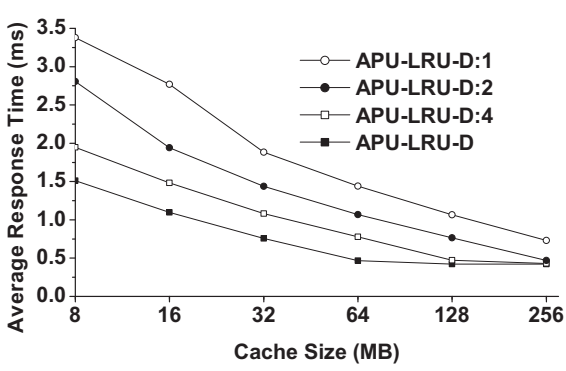

(e) IOzone: 2-way set associative.

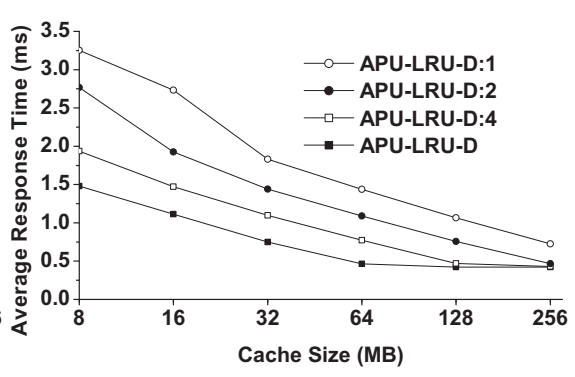

(f) IOzone: 4-way set associative.

Figure 3. Comparison of average response time among various distributed cache configurations.

- APU-LFU: write-back cache with APU applying LFU algorithm. If multiple dirty cache lines share the same access frequency, the LRU policy is applied to choose one cache line among them. Only one cache line is selected each time.

- APU-LRU-D: APU-LRU version with the logically distributed cache. $N$ cache lines are selected so that these cache lines can be written to $N$ flash packages in parallel.

APU-LRU-D: $x(1 \leq x<N)$ : the cache is physically divided such that $x$ flash packages share a single cache.

APU-LFU-D: APU-LFU version with the logically distributed cache.

In the following text, we call the first three types of cache as the centralized cache since they either do not have automatic update or only update one cache line at a time. Correspondingly, the other three cache types are called the distributed cache.

In order to minimize the intervention to the normal operations of the cache, the automatic update mechanism is applied in the background when the SSD device is idle. The cache manager monitors the activity inside the SSD. After a specific amount of SSD idle time, the automatic update action is activated, i.e., the data in the selected dirty cache lines are written into the flash media. By observing the fact that it normally takes $0.3 \mathrm{~ms}$ to write one page data into the flash memory, this idle time is set to 2 $m s$ for all applications in the simulation unless otherwise specified. Once the automatic update process is invoked, it will continue flushing the dirty cache lines to the flash media until a read/write request occurs.
In terms of cache organization, three different settings are simulated, i.e., direct mapped, 2-way set associative and 4way set associative. As for the settings of the flash memory, the internal copy back and the background cleaning features are turned off in the simulation. The size of the SSD is 256 GB in this work unless otherwise specified. The SSD device consists of 8 packages, each of which is composed of 16 planes. Each plane contains 8,192 blocks, each of which consists of 64 pages. The size of a flash page is $4 \mathrm{~KB}$, which is the size of a cache line as well.

We use both the microbenchmark and the application benchmarks to compare the different cache configurations. Since the cache is mainly designed to reduce the response time for write requests, the microbenchmark is a 'random write only' one in which the write address is randomly generated. The request size is randomly set between 0 and 8192 512-byte logical blocks. The total number of requests is $20 \mathrm{~K}$. We selected three applications, IOzone [4], PostMark [5] and Source Control [6], to study the performance of the proposed cache design. Both IOzone and PostMark are standard file system benchmarks. Source Control is the trace file of data access collected on a server. In this work, we have intentionally converted all accesses in the IOzone benchmark into write requests. On the other hand, response to read requests is studied by using the PostMark benchmark, which is a read-dominant benchmark. More precisely, it has a mix of $83 \%$ reads and $17 \%$ writes. Source Control is a trace file recording the data access on a source control server. It consists of more than 1 million data requests, $30 \%$ reads 


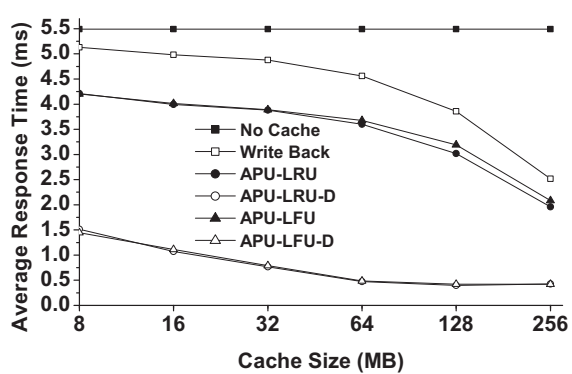

(a) IOzone: direct mapped.

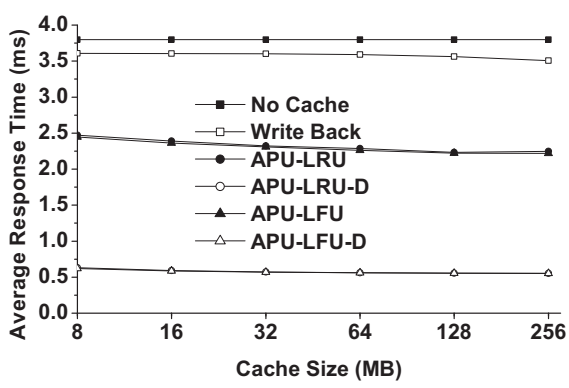

(d) PostMark: direct mapped.

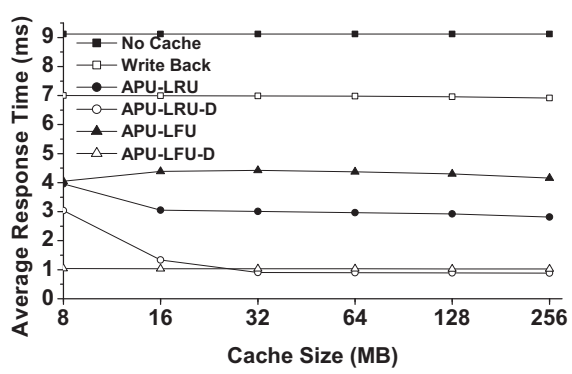

(g) Source Control: direct mapped.

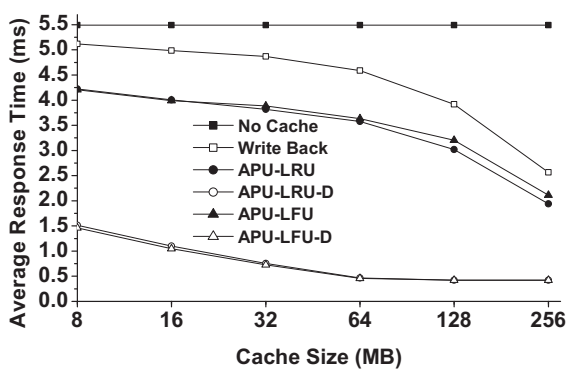

(b) IOzone: 2-way set associative.

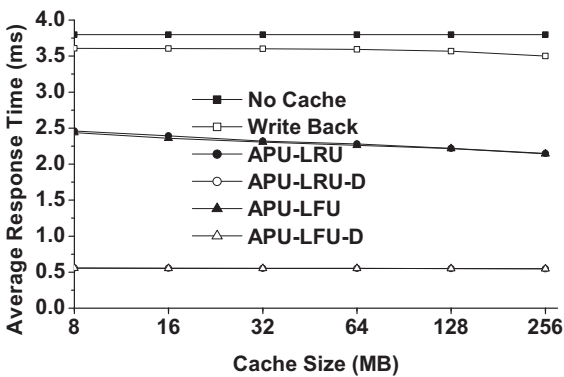

(e) PostMark: 2-way set associative.

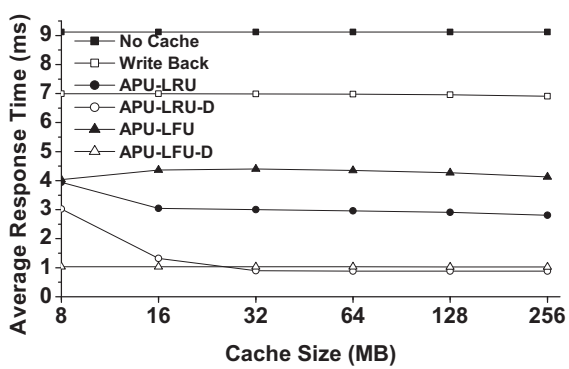

(h) Source Control: 2-way set associative.

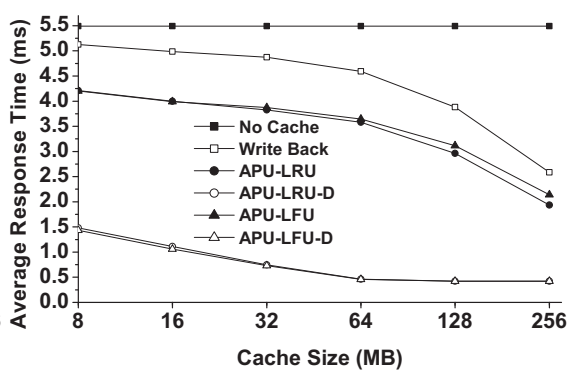

(c) IOzone: 4-way set associative.

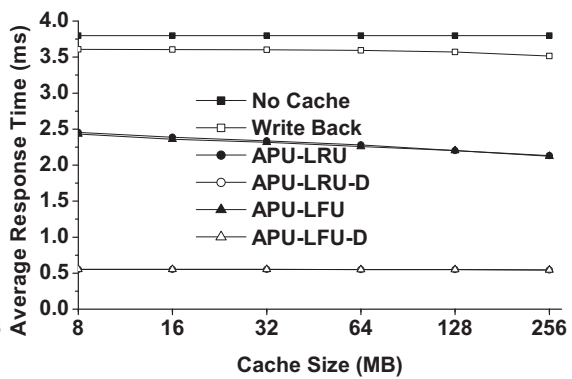

(f) PostMark: 4-way set associative.

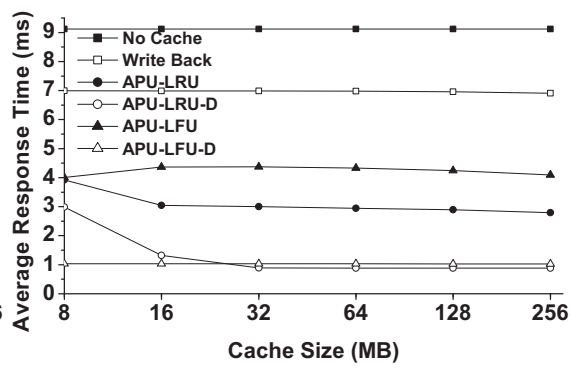

(i) Source Control: 4-way set associative.

Figure 4. Average response time of IOzone, PostMark, and Source Control.

and $70 \%$ writes. The average size of each request for IOzone, PostMark, and Source Control is 445, 720, and 112 512-byte logical blocks, respectively.

\section{B. Comparison of Distributed Cache Configurations}

Figure 2 illustrates various distributed cache configurations. We want to first investigate which configuration will provide the shortest average response time. Therefore, we use two benchmarks, the microbenchmark and the IOZone, to test four different configurations on an 8-package SSD device, i.e., 1:8, 1:4, 1:2, and 1:1. The total size of the cache for different configurations is the same.

The comparison among four different configurations is illustrated in Figure 3. Apparently, the logically distributed cache outperforms all other configurations by a big margin. This advantage is more evident when the overall cache size is comparably small, e.g., $\leq 64 \mathrm{MB}$. Although a physically distributed cache design may reduce the conflict due to the writes belonging to different flash pages, a smaller cache will significantly increase the manual eviction rate. For example, for a direct mapped $8 \mathrm{MB}$ distributed cache, the overall manual eviction count is $1,023,672,1,468,167,3,135,794$, and 4,292,590, respectively, for $1: 8,1: 4,1: 2$, and $1: 1$ (cache:flash package) configurations in the simulation using IOzone benchmark. When the size of the cache increases, the manual eviction rate keeps falling, which contributes to the small difference in terms of average response time between the logically distributed cache and other physically distributed caches for large cache size. Since the logically distributed cache consistently provides the smallest average response time, it is chosen as the design of distributed cache in the following study.

\section{Performance and Lifetime Improvement}

The simulation results of performance are shown in Figure 4 , presenting the average response time of the SSD 
against the cache size, for the different cases considered. It is evident that the inclusion of the proposed cache always leads to a reduction in the access time. Even a simple writeback-only cache can reduce the the average response time by more than $50 \%$ for IOzone benchmark when the cache size is $256 \mathrm{MB}$. However, it is observed that the writeback-only cache can just provide marginally improvement for PostMark benchmark. The limitation of the write-backonly cache justifies the necessity of the automatic periodic update feature. Averagely the centralized cache is capable of reducing the response time by $50 \%$. The logically distributed cache can reduce the response time by a remarkable $90 \%$.

Referring to Figures 4(a) to 4(c), it is seen that the average response time drops for all types of caches as the size of the cache increases. This trend is due to the reduced conflict rate as the cache size increases. When the size of the cache reaches $256 \mathrm{MB}$, even the conflict rate of the write-backonly cache is so low that the performance is approaching the cache with APU-LRU/LFU. For the distributed cache, the effect of parallel cache updating is so evident that even an 8 MB cache can introduce a $73 \%$ drop in response time. The performance of the parallel cache updating is very significant such that a further increase of the cache size beyond $64 \mathrm{MB}$ has almost no effect in the response time.

For the PostMark benchmark, as seen in the results from Figures $4(\mathrm{~d})$ to $4(\mathrm{f})$, the inclusion of the proposed cache reduces the response time by up to $45 \%$ for the centralized cache with automatic update. However, an increase in cache size does not show a significant improvement as compared with the IOzone benchmark for the write-back-only case. This is probably because the access patterns result in cache thrashing with repeated conflicts. This is confirmed by the fact that automatic update gives a significant improvement in performance; as mentioned, the automatic update feature is designed to minimize evictions due to conflict misses.

The results of Source Control benchmark demonstrate interesting phenomena. First, the average response time almost keeps unchanged when the size of the cache is bigger than $16 \mathrm{MB}$. The average response time is $9.12 \mathrm{~ms}$ without the cache, which is reduced to $7 \mathrm{~ms}$ with a write-back cache. This time is further reduced to around $4 \mathrm{~ms}$ and $3 \mathrm{~ms}$ after applying APU-LFU and APU-LRU, respectively. The quite close response time on various cache sizes is contributed by the relatively evenly distributed access addresses of the benchmark. Both APU-LFU-D and APU-LRU-D policies can reduce the average response time to $1 \mathrm{~ms}$, which is only $11 \%$ of the response time without a cache.

The other benefit of the cache is to reduce the number of writes to the flash media, which can increase the life of SSDs. Generally, the bigger the size of the cache is, the more writes can be saved from being written into the flash memory. The write-back-only policy reduces more writes than the APU policies since writes to the flash media are only due to the on-demand updating. For example, number of writes to flash media is reduced by up to $56 \%$ for IOzone benchmark. The reduction of writes to flash media on PostMark and Source Control is $4 \%$ and $10 \%$, respectively. The differences between the write reduction on benchmarks match the ondemand update rate on the cache. For instance, on a $256 \mathrm{MB}$ write-back-only 2-way-associative cache, the eviction rate is $45.5 \%, 96.3 \%$, and $90 \%$ for IOzone, PostMark, and Source Control, respectively. The best performing distributed cache reduces the least number of writes to the flash memory since it is most aggressive in updating dirty cache lines.

\section{CONCLUSIONS}

This paper proposes to use a distributed cache on flashbased solid-state drives to improve performance and lifetime. Various configurations of distributed cache are presented and evaluated. It turns out that the logically distributed cache outperforms all other physically distributed configurations in terms of average response time. Although all the flash packages in a single SSD device share the logically distributed cache for caching the write requests, multiple dirty cache lines are selected simultaneously during the automatic periodic update period so that all flash packages are written at the same time. To evaluate and demonstrate the benefits of the proposed distributed cache, simulations are carried out on synthetic and real-life benchmarks. The results clearly indicate performance improvements with the distributed cache. Improvements in write access time up to $90 \%$ are observed. Additionally the on-board cache is capable of reducing the number of physical writes to the flash media, although the distributed cache generally introduces more writes to the flash media compared with the centralized cache due to its aggressive updating.

\section{REFERENCES}

[1] M. Huang, O. Serres, V. K. Narayana, T. El-Ghazawi, and G. Newby, "Efficient cache design for solid-state drives," in Proc. 7th ACM International Conference on Computing Frontiers (CF'10), May 2010, pp. 41-50.

[2] N. Agrawal, V. Prabhakaran, T. Wobber, J. D. Davis, M. Manasse, and R. Panigrahy, "Design tradeoffs for SSD performance," in Proc. 2008 USENIX Annual Technical Conference, Jun. 2008.

[3] J. S. Bucy, J. Schindler, S. W. Schlosser, and G. R. Ganger, The DiskSim Simulation Environment Version 4.0 Reference Manual, Parallel Data Laboratory, Carnegie Mellon University, May 2008.

[4] IOzone Filesystem Benchmark, http://www.iozone.org.

[5] J. Katcher, PostMark: a new file system benchmark, Technical Report TR3022, Network Appliance, Oct. 1997.

[6] D. Narayanan, A. Donnelly, and A. Rowstron, "Write offloading: Practical power management for enterprise storage," ACM Transactions on Storage, vol. 4, no. 3, pp. 10:1-10:23, Nov. 2008. 